\title{
Percepción de la felicidad en jóvenes universitarios no unidos en pareja, versus, jóvenes no estudiantes universitarios y unidos en pareja
}

\section{Asael Ortiz Lazcano}

Universidad Autónoma del Estado de Hidalgo, Pachuca Hidalgo, México. lazcano@uaeh.edu.mx

\begin{abstract}
Resumen
Esta investigación ofrece resultados de cómo entienden la felicidad los jóvenes de la zona metropolitana de la ciudad de Pachuca, específicamente la percepción de que objeto o concepto es el ancla de su felicidad. La fuente de datos es una encuesta que tiene un grado de confianza del 93 por ciento, con un error de estimación del 8 por ciento a nivel global y una no respuesta del 20\%. Recabó información de 640 hogares útiles seleccionados. Los resultados diferenciados son producto de la comparación de dos grupos de jóvenes, el primero de ellos universitarios que no se encuentran unidos en pareja, para estos las variables de peso para alcanzar la felicidad fueron 1) Haber conseguido cosas importantes que quieren en la vida; 2) la salud; 3) las finanzas; 4) las relaciones afectivas con la familia y 5) su libertad de los jóvenes universitarios. Por otra parte los jóvenes que son unidos en pareja sin importar el tipo de unión, y que no son estudiantes universitarios, anclan su felicidad principalmente en tres variables, el ingreso percibido por su trabajo, la salud y la familia.
\end{abstract}

Palabras clave: Jóvenes unidos, jóvenes universitarios, felicidad, bienestar subjetivo percibido.

\section{Introducción}

La presente investigación pretende analizar la percepción de que hace felices a los jóvenes, entendiendo esta edad entre los 18 y 28 años, para ello se analizarán dos encuestas cuantitativas, apuntaladas desde una perspectiva demográfica. El objetivo que guía la presente investigación se sustenta en lo referido por diversos autores, en donde la posesión de un bien ya sea material o inmaterial, deseado o anhelado por una persona dará la felicidad (Alarcón, 2002). Es muy importante referir que esta postura parece que genera un brete, ya que para algunos lo que es anhelado vehemente, para otros puede incluso no tener ningún valor. De igual forma en esta postura pueden incluirse elementos físicos materiales 
tal como dinero, ropa, autos, viviendas, o elementos no materiales como la salud, hasta cuestiones de religiosidad, de moralidad y por supuesto de ética, al margen de lo que se entienda y desde las diversas aristas que se le analice. Además se pretende investigar dos grupos de personas jóvenes, que por diversas posturas pueden alcanzar de formas diferentes la felicidad, por un lado, jóvenes estudiantes universitarios que no estén unidos en pareja, por otra parte jóvenes no estudiantes unidos, ya sea esta legal o consensual. Este análisis se hace imaginando la situación incardinada en la tradición mexicana, donde los jóvenes a la edad casadera -ya sea que estén dentro de un matrimonio o uniones consensuales- juega un papel preponderante en su realización de la vida adulta, incluso sobre la idea de continuar sus estudios de nivel profesional.

Este proyecto de investigación parte de la definición de felicidad, que prácticamente fue revivido por conceptos de calidad de vida y bienestar, cuyo ideal estaba más allá de los bienes materiales, por ende, generaron la duda y discusión académica resucitando conceptos filosóficos, y sobre todo con aquella intención de medirlos en nuestras sociedades. Hay que diferenciar entre la felicidad, que técnicamente para algunos se llama bienestar subjetivo percibido de lo que es nivel de vida. El bienestar subjetivo percibido o calidad de vida es el disfrute que tienen las personas a partir de su realidad vivencial, es decir, es la delta entre lo que tengo y vivo diariamente, con lo que aspiro. Si esa diferencia es mayúscula, entonces generará infelicidad, por el contrario, si la delta es baja, entonces el sujeto encuentra la felicidad, entiende su vida y su entorno vivencial (López, 2018). Por el contrario, nivel de vida tiene que ver con el dinero, con las posesiones materiales, por ello se entendería que a mayor dinero mayor nivel de vida, aunque no necesariamente mayor felicidad. Sin embargo para muchos puede haber una gran relación y correlación entre estos dos conceptos (López, 2018).

Aristóteles con su libro Ética a Nicómaco, hace mención que el hombre alcanza esa felicidad a partir de que adquiere el bien deseado, por ello debe haber felicidad asociada a ese bien anhelado, pero siempre de la mano de la ética. Para Aristóteles también la felicidad llega cuando hay autosuficiencia, pues parece que el bien completo es autosuficiente, pero la autosuficiencia no la define como la vida en soledad, o vida solitaria. Por ello para Ferrater se refiere al hombre dotado de bienes, que le permite vivir una vida contemplativa, que asociada a la ética, cuenta con los bienes que le dan tranquilidad, paz espiritual y por ende felicidad (Ferrater, 1969).

Para Alarcón los objetos o bienes materiales que promueven la felicidad pueden ser de naturaleza variada y pueden ser éticos, económicos, religiosos, estéticos, sociales, materiales o incluso ideales (Alarcón, 2002). Esta idea la asocia a lo referido por Kant, quien mencionó que el hombre no sabe cómo ser feliz, porque hay muchas formas de alcanzar la felicidad, puede ser a través de cosas materiales, espirituales, de valor monetario, de valor de conocimiento, pretende una larga vida, tener salud, belleza u otros 
bienes u objetos (Kant, 1946). Estos ideales llevan a discutir si en realidad la felicidad está atada a los objetos materiales, Alarcón retomando a Aristóteles refiere que para ser feliz se necesitan bienes exteriores que permitan dedicarse a la vida contemplativa y satisfacer sus propias necesidades. De esta idea plantea, que la felicidad en cierta medida depende de bienes, ya sean materiales o no, pero bienes perseguidos por los seres humanos (Alarcón, 2002).

Lu, Gilmour y Kao (2001) analizó la felicidad de los jóvenes, entre Taiwán e Inglaterra, el trabajo mostró resultados similares, donde los jóvenes de Taiwán observan la felicidad anclada a cuestiones culturales, como es entender el ying y el yang, y ese sentido de equilibrio que permite alcanzar la felicidad. Por el contrario los estudiantes universitarios de Inglaterra, si bien entienden a la felicidad como un estado mental deseable, lo asocian a cuestiones materiales, de esfuerzo y logro individual, enfoque legalista y progreso social y material (Lu, Gilmour y Kao, 2001).

En un trabajo de French y Joseph (1999) realizaron un estudio entre estudiantes universitarios, entre sus hallazgos se encontró una alta correlación entre felicidad y religiosidad (French y Joseph, 1999).

Un estudio realizado por Mogilner, Whillans y Norton (2017) encontraron que hay una relación y correlación alta entre la felicidad y el dinero, pero también debe incluirse el tiempo. Los resultados indicaron que la preocupación de las personas radica en cómo distribuir el tiempo y dinero, en cada grupo de personas, para poder alcanzar en mayor medida ese concepto de felicidad. Luego entonces estarían planteando que el dinero no es automático con respecto a la producción de la felicidad, por el contrario el vector tiempo y dinero, de acuerdo a cómo se aplique, produce felicidad, esto en distintos niveles según sea la combinación y claro, las características de cada grupo (Mogilner, Whillans y Norton, 2017).

Recientemente Grover y Helliwell (2019) parten del supuesto de diversos estudios transversales en las sociedades industriales que han demostrado que los que están casados y los que viven como casados, tienen significativamente mayor satisfacción en la vida que aquellos que son solteros, separados, divorciados o viudos. Incluso algunos estudios utilizando encuestas de panel de países como Alemania y Reino Unido, han sugerido que si bien la satisfacción de vida puede elevarse durante algunos años después de la unión, con el tiempo vuelve a caer a niveles prematrimoniales (Grover y Helliwell, 2019).

Por otra parte Clark y Georgellis (2013) también encontraron que las personas casadas son más felices en los años inmediatamente antes y después de su matrimonio, pero para aquellos que habían estado casadas durante al menos 5 años, la felicidad tiene a disminuir (Clark y Georgellis, 2013). 
Qari (2014) trabajando con jóvenes de Berlín encontró que el uso de los 5 años referidos por Clark y Georgellis, permite calcular la utilidad, pero si se utilizan sólo 1-2 años antes del matrimonio como categoría de referencia, probablemente hay un error, ya que en ese momento se vivía entre los jóvenes un romance, un periodo de enamoramiento, lo que puede generar un aumento en la felicidad reportada. Anteriormente Zimmermann y Easterlin (2006) analizaron datos de una encuesta transversal y encontraron que cuando los individuos permanecen casados dos o más años no vuelven a su valor inicial de felicidad que tuvieron antes del matrimonio (Zimmermann y Easterlin, 2006).

La hipótesis que guio este trabajo, radica en que los jóvenes universitarios no unidos anclaban su felicidad a objetos totalmente diferentes a los jóvenes unidos no universitarios.

\section{Resultados}

\subsection{Descripción del método}

Diseño estadístico de la Encuesta Percepción de la Felicidad en jóvenes de la Zona Metropolitana de la Ciudad de Pachuca Hidalgo 2019. La población objeto de estudio está compuesta por las personas estudiantes de nivel profesional de 18 años o más que habitan en viviendas particulares de la zona metropolitana de la ciudad de Pachuca, no se han incluido a las personas que habitan en viviendas de tipo colectivo, tal como cárceles, hospitales, asilos, conventos, hoteles, casas de huéspedes, instalaciones militares, etcétera. Los municipios incluidos en la muestra fueron, Pachuca, Mineral del Monte, Mineral del Chico, Mineral de la Reforma, Epazoyucan, Zempoala, San Agustín Tlaxiaca y Zapotlán de Juárez, en el Estado de Hidalgo.

El levantamiento de campo de la prueba piloto se realizó en la zona metropolitana de la ciudad de Pachuca Hidalgo en el mes de febrero del año 2019, el número de viviendas que se visitaron para aplicar la encuesta de la prueba piloto fueron 40, la aplicación de la encuesta en las dos zonas fue aceptable y funcional. La realización de la encuesta piloto permitió mejorar el cuestionario y la redacción de algunas preguntas, así como mejorar el entrenamiento de los entrevistadores en la aplicación del cuestionario.

Se utilizó un diseño de muestreo probabilístico multietápico por conglomerados, lo que obligó a corregir la muestra en un $20 \%$ por la no respuesta. La unidad de muestreo de la primera etapa fue municipal, y se continuó de acuerdo a todas las áreas geográficas básicas (AGEBs) existentes.

La encuesta tiene un grado de confianza del 93 por ciento, con un error de estimación del 8 por ciento a nivel global y una no respuesta del 20\%. El trabajo de campo para el 
levantamiento de la encuesta se efectúo en el periodo del $1^{\circ}$ al 25 de febrero del año 2019; el cuestionario estuvo conformado por 15 preguntas que condensa 98 ítems. La encuesta recabó información de 640 hogares útiles seleccionados.

Las exigencias de precisión y confianza anteriores, obligaron a utilizar un esquema de muestreo simple sin reemplazo, con un tamaño de muestra entre 138 y 320 casos para cada dominio, a efectos del diseño se tomó el punto máximo el de 320 para que fuesen representativos para cada sexo y por la diferenciación estudiantes no unidos, así como unidos y no estudiantes Sánchez, 2018; Martínez, 2019). En conclusión, se levantaron dos diferentes encuestas, una para jóvenes alumnos no unidos, y una segunda para jóvenes unidos no alumnos, cada una de ella con la representación por sexo antes mencionada, así como con un total de 320 cuestionarios levantados en cada encuesta. Los municipios que se incluyeron dentro de la muestra son 8 , y en promedio se aplicó el siguiente porcentaje de cuestionarios en cada uno de ellos, Mineral del Monte (2.5\%), Epazoyucan (2.5\%), Mineral de la Reforma (26.2\%), Zapotlán de Juárez (3.1\%), Pachuca de Soto (49.4\%), San Agustín Tlaxiaca (6.3\%), Zempoala (8.1\%) y Mineral del Chico (1.9\%).

\subsection{Resumen de resultados}

Como se ha referido anteriormente se hará la comparación de dos encuestas, la primera jóvenes universitarios no unidos, y una segunda, jóvenes unidos no universitarios, ambas levantadas en la zona metropolitana de la ciudad de Pachuca Hidalgo. Esta comparación según sexo, se genera en razón de los planteamientos teóricos que aducen que los jóvenes casados son más felices (Grover y Helliwell, 2019), mientras que otros como Ahn et al. (2019) ha encontrado que el joven que estudia es más feliz que el joven que trabaja, y refiere la importancia del dinero, ya que los jóvenes que ganan más dinero en sus trabajos son más felices que los jóvenes que ganan menos dinero (Ahn et al., 2019). Se describirán algunos resultados generales para cada una de las encuestas, para después proceder a trabajar con ellas.

Todos los entrevistados ya fueran hombres o mujeres, no vivían unidos en pareja, es decir no estaban casados legalmente, consensualmente, religiosamente, no vivían en concubinato, y tampoco no habían vivido en pareja. De ellos $36.9 \%$ dijeron que trabajaban y estudiaban, ligeramente se observa que en esta situación se ubican un mayor número de hombres que de mujeres. En la economía formal están insertos apenas $15 \%$ de ellos, que reportan tener empleos de ese tipo, una gran mayoría de estos (85\%) lo hace dentro de la economía informal, esto sin duda se explica por el tiempo que deben cubrir no solamente en su trabajo remunerado, también en sus horarios escolares, lo que les impide tener un trabajo formal. Las edades de este grupo oscilaron entre los 18 y 27 años de edad. 
Por lo que hace a los jóvenes unidos y que no estudian actualmente en la universidad, Inicialmente es posible mencionar que un $90.6 \%$ de los hombres refirió que se encuentra laborando, mientras que un $70.3 \%$ de las mujeres también mencionó que trabaja de forma asalariada y que es un porcentaje muy alto. De forma global un $32.7 \%$ trabaja en el mercado formal, mientras que $66.3 \%$ lo hace en el informal, se observa que una mayoría de mujeres están en la formalidad, principalmente en el sector terciario y de servicios. Los hombres se desempeñan más como cuentapropias o empleados, pero dentro de un mercado mayoritariamente informal.

Un dato interesante es que la correlación de Pearson entre la variable ingreso y el nivel de felicidad reportado por los alumnos no unidos es de 0.588 , lo que indica que existe una correlación media, pero que en cierta medida sugiere una correlación aceptable; el chi cuadrado es 0.000 , lo que indica que existe relación entre ambas variables. Por otra parte la correlación de Pearson entre la variable ingreso y el nivel de felicidad reportado por los jóvenes unidos no universitarios es 0.455 , lo que sugiere que existe una correlación media, el chi cuadrado es de 0.000 , lo que indica que existe relación entre ambas variables. Sin embargo parecen más felices los alumnos universitarios solteros que trabajan, que los jóvenes unidos que trabajan, aunque porcentualmente obtengan mejores ingresos.

También existe otro dato interesante por comparar, hablando de los jóvenes universitarios solteros es que aquellos que no trabajan reportan un promedio de 8.43 de felicidad, mientras que los que sí trabajan asalariadamente alcanzan en promedio 7.55 de felicidad, tal vez se deba a la doble carga de trabajo que deben enfrentar los jóvenes que estudian y trabajan.

Hablando de los jóvenes no universitarios que viven en pareja y que trabajan asalariadamente se autocalifican con un promedio de 8.55 de felicidad, mientras que los jóvenes de ese mismo grupo que no laboran alcanzan un 8.20 de felicidad, diferenciando según sexo, ligeramente se reportan más felices los hombres que no trabajan con 8.27, mientras que las mujeres alcanzan un 8.13 de felicidad.

Para dar inicio a las preguntas que tienen que ver con la percepción de la felicidad, todas se construyeron de forma Likert, por ello es factible obtener un alfa de Cronbach para que nos indique el grado de fiabilidad del instrumento aplicado, para la encuesta de los jóvenes universitarios no unidos el alfa de Cronbach alcanza 0.819 , lo que se traduce como un buen indicador que valida la correcta construcción del cuestionario.

Por lo que hace a la encuesta de jóvenes unidos no universitarios evaluando el alfa de Cronbach fue de 0.859 , lo que se traduce como un buen indicador que también valida la correcta construcción del cuestionario 
Al analizar estas preguntas se advierten diferencias importantes entres el grupo de universitarios solteros no unidos, y el grupo de jóvenes unidos no universitarios. Al preguntar si en la mayoría de los sentidos, su vida se acerca a su ideal, destaca como dos de cada tres universitarios estuvo de acuerdo, mientras que solo uno de cada tres pensó en ese sentido de los jóvenes unidos no universitarios. Al cuestionar sobre si las condiciones de su vida son excelentes, nuevamente seis de cada diez universitarios dijeron que era así, mientras que tres de cada diez no universitarios pensaron de esa forma, sin duda las responsabilidades de familia, las necesidades alimentarias, de vivienda, salud, entre otras, sugieren que la forma de observar la vida es totalmente diferente.

A los universitarios las cosas que más refieren les han causado satisfacción en el último mes son, su instituto, escuela o facultad (81.6\%), su desempeño en la escuela (83.5\%), su salud $(84.4 \%)$, sus amigos $(85 \%)$, sus relaciones afectivas con la familia $(85.7 \%)$, su desempeño en el hogar (86.6\%), su vivienda (87.6\%) y su libertad (88.4\%). Lo que menos les causa satisfacción es la situación de su país (36.6\%), su seguridad (57.8\%) y la seguridad de los suyos (58.1\%).

Los jóvenes no universitarios difieren un poco, consideran que los elementos que les brindan mayor felicidad son la salud (80.8\%), su desempeño en el hogar $(80.8 \%)$, sus relaciones afectivas de pareja $(81.6 \%)$ y sus relaciones afectivas de familia (93.4\%). Las situaciones con menor puntaje fueron la situación de su país (15.4\%), su futuro (29.8\%) y sus finanzas $(34.3 \%)$.

\subsection{Conclusiones}

Dentro de los resultados de la presente investigación, se concluye que la felicidad de los jóvenes universitarios solteros y los jóvenes no universitarios unidos en pareja, está anclada en una parte importante de la estabilidad económica de los primeros, y el ingreso percibido de los segundos, esto es a mayor dinero mayor felicidad. Un dato complementario es que no existe una diferencia significativa de declararse feliz, a partir de ser estudiante universitario soltero, o por estar unido si ser estudiante, la percepción de la felicidad es muy similar, 0.4 puntos hace la diferencia en favor de los jóvenes unidos no universitarios.

De igual forma los objetos o bienes materiales que promueven la felicidad pueden ser de naturaleza variada, aunque para los jóvenes de la zona metropolitana de Pachuca está anclado al dinero de forma importante, corroborando la conclusión anterior. Incluso, se parecen en ese sentido a jóvenes de otras latitudes como el caso de Irlanda, donde las personas tienen infelicidad por no contar con dinero, con amigos, familiares u otros conocidos, y que si tienen esos elementos entonces serán felices.

Es de mencionar que para los dos grupos analizados la religión no jugó un papel importante en la felicidad. Un dato que merece ser mencionado fue al momento de preguntar sobre los 
principales problemas que observan a municipal, los jóvenes universitarios mencionaron la pobreza $(13.1 \%)$, el desempleo $(24.3 \%)$ y el deterioro ambiental con $24.3 \%$; mientras que los jóvenes unidos no universitarios refirieron la violencia $(11.0 \%)$, la pobreza (19.8\%) y el desempleo $(36.9 \%)$, aquí destaca que el deterioro ambiental nunca fue referido por el segundo grupo, lo que sugiere que se desarrollan en contexto diferenciados y los jóvenes universitarios lo aprenden a partir de todas las discusiones que se dan en torno a la importancia del cuidado del planeta y del ambiente que nos rodea.

Por otra parte la regresión logística binomial aplicada en jóvenes universitarios solteros, muestra que las variables de peso que explican la felicidad en ellos, está el sentir que han conseguido cosas importantes en su vida hasta ahora, otra variable importante es la salud, sus finanzas son muy importantes, aunque dependan en gran parte de los padres, otro punto es que haya buenas las relaciones afectivas con la familia, así como su libertad. Por lo que hace a los jóvenes unidos en pareja no universitarios explican la felicidad a través de tres variables, el ingreso en sus empleos, la salud y las buenas relaciones afectivas con la familia.

\section{Referencias}

Alarcón, R. (2002). Fuentes de la felicidad: ¿qué hace feliz a la agente? Revista de Psicología de la Pontificia Universidad Católica de Perú (PUCP) Volumen XX, 2, 2002. 169-196. Recuperado de http://revistas.pucp.edu.pe/index.php/psicologia/article/view/3710/3692

Alarcón, R. (2014). Funcionamiento familiar y sus relaciones con la felicidad. Revista peruana de psicología y trabajo social 3(1), 61-74. Recuperado de http://revistas.uigv.edu.pe/index.php/psicologia/article/view/55/62

Ahn, N. Mochon, M. F. y De Juan, R. (2011). La felicidad de los jóvenes. Papers 2012, 97/2, págs. 407-430. Recuperado de https://papers.uab.cat/article/view/v97-n2-mchon-anh-de_juan/pdf

Clark, A. \& Georgellis, Y. (2012) Back to baseline in Britain: adaptation in the British Household Panel Survey. Economica, 2013, vol. 80, número 319, 496-512, Recuperado de https://ideas.repec.org/a/bla/econom/v80y2013i319p496-512.html

Ferrater, M. J. (1969). Diccionario de filosofía (Tomo 1). Buenos Aires: Editorial Sudamericana.

French S. and Joseph, S. (1999). Religiosity and its association with happiness, purpose in life, and self-actualization, Journal Mental Health, Religion \& Culture, 2:2, 117-120, Volume 2, 1999 doi:10.1080/13674679908406340

Garduño, E. L. Salinas, A. B. y Rojas, H. M. (2005). Calidad de vida y bienestar subjetivo en México. México D.F.: Plaza y Valdés.

Grover S. and Helliwell, J. F. (2019). How's life at home? New evidence on marriage and the set point for happiness. J Happiness Stud 20: 373-390. doi.org/10.1007/s10902-017-9941-3 
Gutiérrez, S. R. (2006). Historia de las doctrinas filosóficas. México D.F.: Editorial Esfinge.

Kant, M. (1946). Fundamentación de la metafísica de las costumbres. Buenos Aires: Espasa.

López, R. D. (2018). Emoción y sentimientos. No somos seres racionales, somos seres emocionales que razonan. Buenos Aires: Ariel.

Lu, L., Gilmour, R. and Kao, S.F. (2001). Cultural valuesand happiness: an east-west dialogue. Journal of social psychology, 141, 477-493.

Martínez, B. C. (2019). Estadística y muestreo. Bogotá, Colombia; ECOE Ediciones.

Mogilner, C. Whillans, A. and Norton, M. I. (2017). Time, money, and subjective well-being in E.Diener, E. Oishi, \& L.

Sánchez, C. J. J. (2018). Errores de muestreo. Precisión de los estimadores en encuetas probabilísticas. Madrid España:Dextra Editorial.

Zimmermann, A. \& Easterlin, R. (2006). Happily ever after? Cohabitation, marriage, divorce, and happiness in Germany. Population and Development Review 32(3), 511-528. doi.org/10.1111/j.1728-4457.2006.00135.x 